\title{
The sound of a spherical cow
}

\author{
Julian Bradfield \\ University of Edinburgh
}

\begin{abstract}
I consider the use of computational simulations in phonology, and the benefits and dangers of making abstractions, or failing to make abstractions. I argue that the potential of simulation studies is not yet realized as it could be.
\end{abstract}

\section{Introduction}

Phonology, and grammar in general, has been viewed in discrete computational terms since at least Panini, whose grammar of Sanskrit is effectively a formal re-write system (Ingerman 1967). With the ease of modern programming environments and our fast personal computers, every theoretical phonologist can now implement and test their theories - as Karttunen (2006) demonstrated, even quite simple theories may not actually do what their author intended. Such uses of computation raise no new problems for phonology or phonologists in the generative tradition; they merely let us do more of what we have done for two millennia, and faster.

During the twentieth century, increasing ease and power of computation also encouraged the use of simulation studies in phonology (and language more generally) such as the use of continuous parameters in vowel phonology; the use of probabilistic and stochastic models; models of phonological change based on agents; models of phonological learning in the individual. Such models may not have deterministic, repeatable results, and may not even have results easily amenable to statistical analysis; and the techniques required for analytical study may be quite mathematically sophisticated (such as the construction and numerical solution of sets of partial differential equations). When building simulations, there are many choices to be made about how much, and in which aspects, to abstract away from details of reality, and the effect of different abstractions and simplifications may not be clear. The consequence is that many simulation studies show, at best, that theory $X$ is at least a possible account of phenomenon $Y$.

More ambitiously, if simulations are designed with careful analysis of the underlying theories, analyses of the sources of error, and the rest of the apparatus usual in physical and engineering science simulation studies, and then simulations are conducted over a wide range of possible configurations and parameter settings, one might say with some confidence what is not possible, as well as what is possible; and one may even produce numerical results, testable for the degree of agreement with empirical data.

\footnotetext{
* I thank Bart de Boer, Paul Boersma, Kateřina Chládková and James Kirby for discussions and comments over the years, as well as attenders at the Phonetic Universals conference in Leipzig in 2010 and the 19th Manchester Phonology Meeting in 2011, where the first versions of the work of sections 3.3 and 4.2 were presented. I thank the anonymous reviewers for careful and expert commentary on the paper.
} 
This article considers some case studies of the use of computational simulations, and simulations of computational theories, in phonology, critically examines what conclusions can be drawn from them, and discusses new simulations inspired by this examination.

The title comes from a long folklore tradition of jokes about physicists and applied mathematicians.

$Q$ : How does a physicist milk a cow? A: Consider a spherical cow ...

\section{Models and modelling}

Science (and indeed pre-scientific explanations of the world) is fundamentally a process of constructing more or less abstracted models of reality, positing entities (atoms, quarks, thunder gods) whose existence explains something, and (once thinking becomes scientific) making predictions from the model and validating them against reality.

There is a large literature on the notion of models and modelling in science - Frigg and Hartmann (2017) provides an overview and pointers particularly into the philosophical literature. Space does not permit much discussion of this, but there are some (fuzzy) dichotomies of models that we will mention.

Marr (1977) proposed a dichotomy between type I problems, where it is possible to construct a simplified or abstracted model and compute with it, such as the ideal gas model of gases, or the phonemic theory of sound systems, and type II problems, where there is no solution simpler than the problem itself. His example of the latter was protein folding, which was then and is still insoluble by any means other than a detailed simulation of atomic interactions. In the discrete computational world, the halting problem is a type II problem: the only general way to see if a program terminates is to run it until it does. It often appears that the agent-based models I discuss here are themselves type II problems to solve, as they involve interactions of many processes; however, it is sometimes possible to abstract and approximate, as mentioned above.

Another distinction, commonly made in the computer science modelling literature, is that between discrete models and continuous or hybrid models. Any model involving real numbers cannot be stepwise simulated exactly, and issues of rounding and precision arise; on the other hand, techniques of mathematical analysis can make exact statements about entire regions of the model space in a way that is less common in discrete models. Most phonological simulation models are hybrid (discrete state plus continuous variables), and while there is a large literature on analysis of such models, particularly in computer science, the techniques are non-trivial.

Finally, I should say that I will use the term abstraction in a narrow sense of intentionally removing detail, rather than in the broad sense of mathematizing reality. (Thus, in the broad sense, the Standard Model, like every model, is an abstract model, but in the narrow sense it is not, as despite its known incompleteness, the model does not intentionally omit any detail. On the other hand, a simple harmonic oscillator model of a pendulum does intentionally omit real details.)

\section{The case of vowel systems}

The first case study is the evolution of vowel systems, which provides examples of several dangers and difficulties that arise in the process of constructing models, and drawing contentful conclusions from them. 


\subsection{Early models - dynamical systems}

Modelling of vowel systems goes back to the early days of modern computing, when Liljencrants and Lindblom (1972) placed points in a 3D formant frequency space, imposed the constraint that points should try to 'contrast' (i.e. be as far apart as possible in perceptual space), and simulated the resulting dynamical system. The resulting arrangements of points achieved a reasonable level of match - judged impressionistically - with real vowel systems, although with a number of discrepancies: for example, predicting [i] rather than the more frequent $[\varnothing]$ in seven- and eight-vowel systems. The conclusion was that 'contrast' is an important constraint determining the shape of human vowel systems.

This early model already demonstrates the importance of a key factor in all mathematical modelling of real-world systems: how abstract shall we be, and why are we making various abstractions, and does the resulting loss of fidelity in the modelling vitiate the conclusions? Is it safe to assume a spherical cow? The authors made several abstractions that are repeated in the later work discusssed subsequently. I list some of them here, together with prima facie criticisms, which I examine more carefully in what follows.

(1) Vowels were represented by the first three formant frequencies. Despite debates on formant vs whole-spectrum perception, the issues of speaker variation, etc. (see e.g. Johnson 2005), there is, and was, enough evidence for the key role of formants in vowel perception that this decision was and is likely to be accepted by most.

(2) The boundaries of vowel space were defined by constructing (Lindblom and Sundberg 1969) a model of the vocal tract and basic articulators, to match 'a typical male speaker', and computing the possible formant frequencies emitted by this model. This is not justified over the alternative of using data from actual speakers; it was perhaps simply easier at that time to use their existing model to generate extremes, than to use humans.

(3) The acoustic vowel space is mapped to a perceptual space by converting hertz to mels. The justification is that mels are by definition constructed to match the response of the human auditory system, and this should also apply to our perception of formants. However, over the range of frequencies that formants take, the non-linearity of the mel scale is not very marked, so it is not clear that this step had any significant effect on the results. (There was, indeed, existing work (Stevens 1952) suggesting that a linear scale might anyway have been better.)

(4) The authors need a measure of how widely dispersed - how contrastive - a set of vowels is, in the perceptual space. They choose the measure $-\sum_{i, j: j<i} 1 / r_{i j}^{2}$, where $r_{i j}$ is the Euclidean distance between vowels $i$ and $j$. This is justified by analogy with physics, where many problems about bodies interacting via forces are solved by minimizing the potential energy induced by the forces. In fact, their analogy fails, since the potential energy is the integral of the forces - contrary to their stated intention, they are using a $1 / r^{3}$ force, penalizing close-by vowels very strongly. It is not discussed whether the choice of a $1 / r$ measure instead of a $1 / r^{2}$ measure would have a significant effect.

(5) 'To make computations somewhat easier', the 3-D space is projected down to a 2-D space using $F_{1}$ and a linear combination of $F_{2}$ and $F_{3}$ (still in mel space). On the face of it, this should make a dramatic change: confined to a (2-D) circle, the minimum energy configuration of four points is a square, whereas confined to a (3-D) sphere, it is a tetrahedron, which might project to a quadrilateral, or to a triangle with an internal point, depending on the projection.

(6) Finally, the results of letting this system evolve until it appears to have reached equilibrium are compared with the distribution of real-world vowel systems. However, these systems 
are of course generally reported only at the coarse level of one or maybe a half division on the standard IPA vowel chart. Matching of the 'predicted' system to real systems is impressionistic.

This short and incomplete list illustrates several of the reasons for making abstractions, and also several of the dangers. (1) is a simplification of complex reality, but with good evidence that the simplification should preserve behaviour. (2) and (3) are cases where a more detailed realization is chosen for the sake of matching 'reality' better, but without an argument that the increased fidelity is needed. (4) shows a poorly motivated choice for what may or may not be a critical function needed by the model. (5) shows an simplification purely to make the problem more tractable, with some justification for psychological reality, but one that may change considerably the behaviour of the system. (6) demonstrates the difficulty of comparing even a very abstracted mathematical model with data obtained by others for their own purposes of descriptive linguistics.

The model is conceptually simple, and aims simply to demonstrate that human vowel systems try to maximize contrast (learners of Danish may be forgiven scepticism). In fact, it is sufficiently conceptually simple that the entire numerical simulation set-up is unnecessary. One can reason analytically that given a $1 / r^{2}$ potential (and indeed a $1 / r$ potential, or even any potential correponding to a repulsive force, which shows that their accidental mismatch with physics was not important), and the convex boundary shape, that as the number of vowels increases, they will distribute around the boundary, until the centre becomes nearer to an edge than the distance between vowels on the edge. At this point, centralized vowels appear, and typically there will be many minimal configurations - in a circular space, this happens when the sixth and seventh vowels are added. Which one their (deterministic) simulation arrived at, will have depended on arbitrary factors of precision and rounding. With modern computing power, variation due to such factors is easy to explore; with the resources of 1970, it was not their calculations ran overnight, which would now take a fraction of a second.

It is, then, not clear that the entire paper says much more than "if vowels are subject to a pressure to contrast, they will be arranged to maximize contrast", which is almost a truism. More importantly, the reverse implication, which is what the paper is aiming to establish, does not follow. To show what was claimed, the authors would need to have shown that if there is no pressure to contrast, then human vowel systems do not arise. It is trivial that with neither contrast nor any other constraint, their model would allow random vowel systems; but it is not trivial to show that there is no other constraint than contrast which might lead to the human configurations.

I do not, of course, doubt that maximising contrast is a major factor in the shape of vowel systems; but Liljencrants and Lindblom (1972) provided no substantive support for that belief, which rested quite adequately on informal considerations of communicative efficiency.

\subsection{An agent model}

As modern computing developed, it became possible to make effective use of models based on many interacting particles (e.g. nuclei) or elements (e.g. cells of atmosphere, or portions of an aircraft wing). The social sciences were quick to experiment with such models, with linguistic simulations appearing as early as Klein 1966. In such models, the term 'agent' refers to a process modelling some kind of human (or animal) actor, and which typically interacts with many other agents all running independently. In this subsection, I consider one of the best-known fairly recent phonological examples, again looking at vowel systems.

De Boer (2001) created a model, not of abstract synchronic vowel systems where one is looking for a defined 'optimal' system, but of speakers transmitting vowel systems through 
the generations. The basic framework is the 'imitation game' as described by Steels (1997), which is an instantiation of a framework going back at least to the early 20th century work in population genetics. As applied by De Boer, the key points, and key abstractions are:

(7) A population consists of a number of agents.

(8) Each agent possesses an inventory of 'vowels', identified as points in a 3-D 'articulatory' space modelling height, backness and rounding.

(9) Agents interact by events in which

(a) $A$ chooses a vowel $v$ from its inventory;

(b) $A$ 'says $v$ to $B$ ': transforms $v$ into a 3-D 'acoustic' space (modelling the first three formants), and transmits it to $B$;

(c) $B$ transforms the acoustic signal to a 3-D 'perceptual' space (essentially formants modified by mel and other psycho-acoustic results), and matches it against $B$ 's own inventory (by 'saying its vowels to itself' and seeing which is the best match, according to the Euclidean distance in perceptual space), identifying a vowel $v^{\prime}$;

(d) $B$ says $v^{\prime}$ back to $A$;

(e) $A$ matches $v^{\prime}$ against its own inventory, and signals 'extra-linguistically' to $B$ whether it heard $v$, the vowel it first sent;

(f) if there was a match, $B$ marks a successful communication against $v^{\prime}$, and moves $v^{\prime}$ somewhat in the direction of $v$ (that is, reinforcement learning); if there was no match, $B$ marks a failure against $v^{\prime}$;

(10) from time to time, agents spontaneously add a new randomly situated vowel to their inventory, remove repeatedly unsuccessful vowels, and merge vowels that are perceptually 'too close' together;

(11) some random noise is added at all places where it can be: position of $v$, and tranformations between spaces.

Given this setting, a population of a dozen or two agents is allowed to interact for a few hundred or thousand transactions, and then one examines the inventories of the agents to see how well they match human systems - by impressionistic comparison of pictures. It is claimed that plausibly human-looking vowel systems emerge from this procedure.

Following the discussion in the previous subsection, it will be apparent that this work also contains some decisions that might be 'bad abstractions', or 'bad non-abstractions', as well as good abstractions or good non-abstractions (by non-abstraction, I mean a deliberate decision to retain some detail). Having the space of a thesis, De Boer does discuss some of these issues at some length, though without (in my view) complete success. (See also the review Donegan 2004 for a critical view.) One of De Boer's most interesting sections occurs only in the original thesis (De Boer 1999, §3.1): he first tried to use a fairly concrete articulatory model, similar to that of Liljencrants and Lindblom 1972, to generate not only boundaries of the vowel space but also the formant values. Moreover, he tried to model consonants as well, and distinctive feature phonology. This (hugely ambitious) project resulted in sound systems with 'no relevance whatsoever to understanding human sound systems', and he scaled his ambitions back to the simpler model outlined above. Why did the first model fail? I am confident it was because of the uncontrollable interactions of bad abstractions and bad non-abstractions.

De Boer 2001 has been influential. It also sparked my own interest in simulations in phonology, and consequently I decided to examine it more closely, by re-implementing and attempting to tease apart which abstractions and non-abstractions were good or bad. This I discuss in the following subsections. 

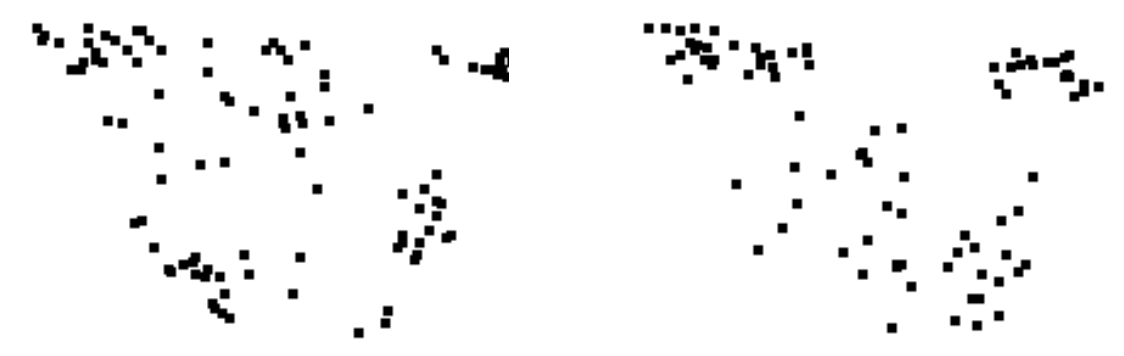

Fig. 1. Two runs of De Boer's (2001) algorithm, with different parameters

\subsection{Detail, parameters and abstraction in simulation}

I re-implemented the framework of De Boer 2001 in Java. Thanks to De Boer's careful descriptions, it was for the most part straightforward.

One aspect of simulation models that I have not yet mentioned explicitly is the role of parameters. Almost every model will end up containing a number of numerical values or functions which encode choices not determined by the underlying theory. In the Liljencrants and Lindblom 1972 model, examples include the strength of the contrast measure, the details of the projection from 3-D to 2-D, the precise details of the numerical hill-climbing algorithm, and so on. De Boer was unusually careful about mentioning these, but nonetheless missed one (at least; I added only those parameters essential for the model). There are a dozen parameters, and there is no really principled choice of value for most of them.

Figure 1 shows two example runs of my implementation of De Boer's (2001) original model, plotting the vowels of 20 agents, each starting with a single random vowel, after a few thousand interactions. In the left-hand picture, 'standard' (copied from De Boer) parameter settings have been used. The community has more or less converged on a five-vowel / $i$, $\mathrm{a}, \dot{\mathrm{i}} / \mathrm{\partial}, \mathrm{o}, \mathrm{u} / \mathrm{system}$. For the particular parameter settings used, four or five vowels are typical; by tweaking parameters, fewer or more vowels can be induced to emerge. However, in the right-hand picture, one parameter has been increased a little: artEpsilon, which governs the amount by which successful listeners accommodate their vowel to what they heard. Here, there are reasonably clear $/ \mathrm{i}, \mathrm{u} /$, but no convergence in the lower half of the vowel space, and indeed more detailed inspection shows speakers with anywhere from four to ten vowels in their inventory.

This set-up with its repeated, extensively randomised interactions is not easily amenable to direct analysis in the way that Liljencrants and Lindblom 1972 was. It is possible that hybrid system techniques or continuous approximation techniques could be applied, but nobody has done so. It has been treated as a type II model, whether or not it is one. Despite this, and although De Boer was explicit in avoiding the teleological 'contrast-maximizing' inherent in Liljencrants and Lindblom 1972, we might still ask whether we learn anything. The explicit biases in the model are to converge with one's peers, and to maintain the identity of phonemes until they become too close together. Implicitly, however, the convergence together with deletion and merging, have fairly directly the effect of biasing toward contrast. As for the shape of the vowel systems, that these match (somewhat) with human vowel systems is a simple consequence of contrast being a function of distance in the vowel space, which makes vowels disperse just as much as the explicit potential function of Liljencrants and Lindblom 1972. In some ways, this model would be more interesting if it failed. 


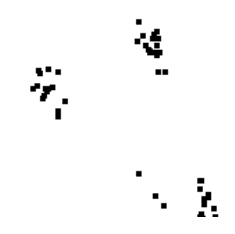

Fig. 2. A run of the simplified model

\subsection{When are details necessary?}

Having established the original model, we can ask which of its details are necessary to the results. In particular, the model has a fairly detailed model of transformations between (pseudo-) articulatory, acoustic and perceptual space. Is this detail of any importance? That is, when we've modelled a basically spherical cow, is there any point in modelling its several stomachs, rather than just having one tube? To investigate this, I produced a simplified version of the model. I threw away almost all the phonetic detail: vowels became simply points inside the unit height/backness/rounding cube, and are transmitted to the listener with no change other than the addition of noise. The only concession was to compress the perceptual front-back dimension of lower vowels, and reduce the size of the rounding dimension, to match the shape of the usual vowel chart, and so make merging more likely in low vowels.

Figure 2 shows a run of the simplified system; parameter settings are mostly the same as in Figure 1(left), save that the perceptual parameter has been adjusted for the new space. One can see that this population has mostly settled on a five vowel system $/ i, \varepsilon, a, \jmath, u /$ that is both more tightly grouped and more human-looking than that in Figure 1 (though there's a noticeable [a-a] split in the /a/ vowel).

The sceptical reader will for some time have been asking a rarely addressed question: are these pictures cherry-picked? Let me be honest. Of course they are, as they almost certainly are in every simulation study ever published (apart from those who do carefully present composite data on many simulations). However, they are reasonably representative of typical runs, judged impressionistically. The sufficiently interested reader can run the programs to see - source code is available in the supplementary materials.

With more experimentation, it turns out that, at least to my eyes, the simplified model produces 'more human' vowel systems not just for five-vowel systems, but for higher numbers of vowels also. It is tempting to conclude, therefore, that De Boer's fairly detailed articulatoryacoustic-perceptual model was a 'bad non-abstraction' - retaining fidelity to reality gives less persuasive results. This is a somewhat disturbing conclusion, as indeed De Boer must have been disturbed when his first, ambitiously detailed, model, gave very poor results. We must hope that the cause lies in inaccuracy of the details, rather than in the decision to retain details.

However, an alternative to being disturbed is to ask whether we can learn something from the more abstract solution giving a better fit. This leads on to my second case study.

\section{Inferring abstractions from simulation}

\subsection{Learning prototypes or features?}

Boersma and Chládková (2010) were interested in whether people learn vowels as prototypes - that is, /e/ is a point in phonetic space which identifies the 'best' /e/ - or whether it can 

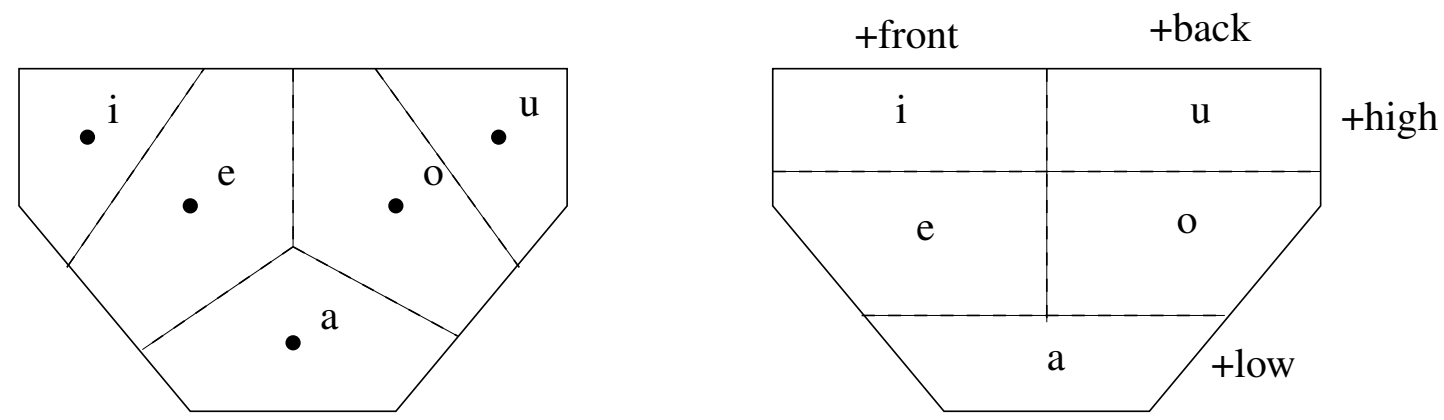
(2010)

Fig. 3. Vowel perception boundaries in prototype and feature models, after Boersma and Chládková
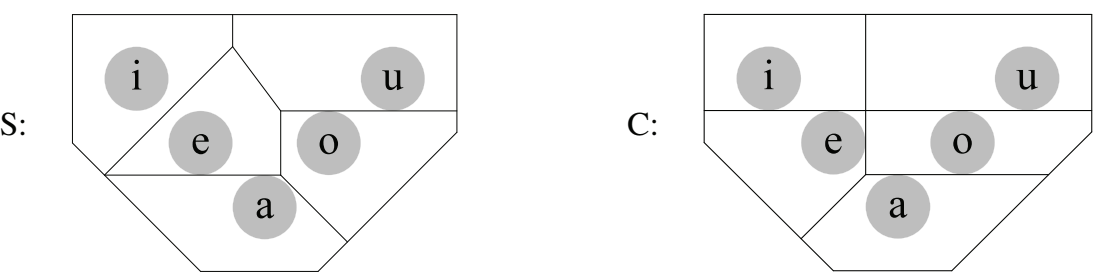

Fig. 4. Predicted Spanish and Czech boundaries, from Boersma and Chládková 2010 [permissions required]

be argued that people learn to discriminate categorical features, so that /e/ is indeed a feature bundle.

Boersma has for some years been developing a sophisticated phonological grammar, one slogan of which is 'bidirectionality': the grammar is layered into modules, which feed up and down to each other. The grammar is constraint-based, in the style of Optimality Theory (Prince and Smolensky 1993), though some of the constraints used are rather different from those of typical OT phonology: for example, so-called 'cue constraints' allow the specification of a vowel by weighting its formants against a large number of points on the frequency spectrum, and thereby allow vowels to be modelled as diffuse, distributional entities rather than points.

Boersma and Chládková (2010) set up a simulation framework in this grammar, with agents learning a 5-vowel system, by re-weighting constraints on the basis of input. They said that when they simulate learners who are learning prototypes, the perceptual boundaries of the agents (as determined by testing them on an array of vowels) are drawn 'diagonally'. On the other hand, if the learners are learning to associate vowels with [ \pm high, \pm back, etc.], then the boundaries are horizontal and vertical. This is illustrated schematically in Figure 3.

Savela (2009) carried out extensive experiments testing the perceptual boundaries of vowels for speakers of many different languages, using artificial stimuli. The results appear to support horizontal/vertical divisions, rather than diagonal divisions. Thus, Boersma and Chládková say, their simulations support the claim that people learn features, not prototypes.

There is also a more specific claim about two languages. Spanish and Czech both have standard five-vowel systems. It is, however, claimed that Spanish phonology makes /a, e/ [central], while in Czech /a/ is [back] and /e/ [front]. Running simulations learning these feature specifications predicts different perceptual boundaries, showing in Figure 4. Boersma and Chládková conducted perception studies on Czech and Spanish speakers, and their results matched Figure 4. (Clearly, they are assuming ternary height and backness features.) 

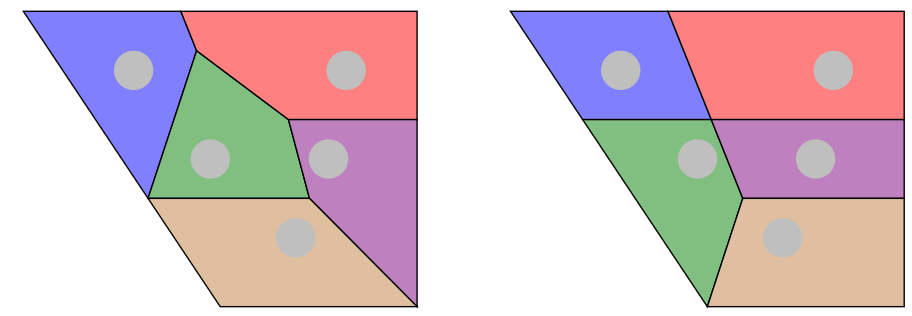

Reinterpretation of prototype vs feature learning expectations of perceptual regions, on the standard vowel quadrilateral. Regions show perceptual regions after training. Grey blobs show centre of distributions used in training.

Fig. 5. Re-drawn results of Boersma and Chládková 2010

\subsection{Analysis and response}

We can make two criticisms of the argument, both of which echo criticisms we made above of Liljencrants and Lindblom 1972. The first is that the simulations add nothing to the argument. If agents model vowels as prototypical points, and incoming vowels are matched by Euclidean distance to the nearest such point, the boundaries in Figure 3(left) are natural. Similarly, if they learn features that are defined to be horizontal and vertical divisions of vowel space, the boundaries in Figure 3(right) are natural. The second is that even granting the argument that the empirical results are consistent with feature learning, there might be other plausible explanations that would give similar empirical results. In particular, could a different 'prototype' learning model account for the facts? I explored the latter by refining the simplified De Boer model I introduced above. Recall that in this model, vowels were essentially points in abstract vowel space, with no subtleties of articulation or acoustics, and a minor adaptation for perception to compress low vowels. To explore the question, I made two changes to the model:

(12) Because we wish to study the evolution of the realization of a stable vowel system, rather than the development of vowel systems, I split the population into adults (whose systems do not change) and children, who learn their vowels by interaction with adults according to the imitation game. As adults age and die, children replace them to maintain the population, and then become adults.

(13) I enriched the notion of vowel, loosely inspired by exemplar theory (Pierrehumbert 2002). However, rather than exemplar clouds for words, or even phonemes, I proposed a simple model in which for a vowel $v$, agents have an articulatory prototype for $v$, but also a convex polygonal region of space encompassing vowels they have heard and recognized as $v$ by nearest matching. The prototype is nudged by the examples they hear; but the perceptual recognition region only expands.

(14) I also ignored the rounding dimension for this simulation.

I also went back to the literature to investigate Spanish and Czech vowels (Harmegnes and Poch-Olivé 1992; Chládková, Escudero, and Boersma 2008; Volín and Studenovský 2007; Ekštein 2004). I did not find strong support for the claim that Czech /a/ is [back], and some argue it is [central] (Aleš Bičan, p.c.). I did, however, find that a typical realization of Czech /a/ is somewhat backer than Spanish /a/, and that a typical Spanish /e/ is higher than a Czech /e/, and the participants in Savela 2009 showed a similar slight difference in their judgement of the 'best' examples. Figure 6 shows schematically perceptual boundaries and best examples from Savela 2009, and Figure 5 shows Figure 4 adapted to the usual vowel quadrilateral.

I converted average formant data from the sources to positions on the abstract vowel chart, and used these positions to seed the articulatory prototypes for 'Czech' and 'Spanish' populations. After running the simulation for a few generations, I measured the perceptual spaces of the agents. The results, together with the original prototypes, are shown in Figure 7. 
Spanish

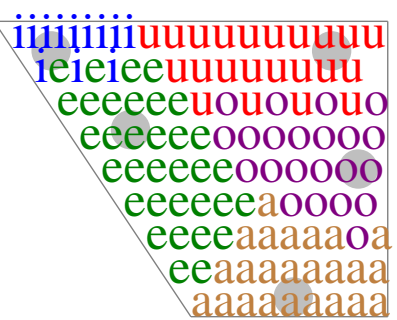

Czech

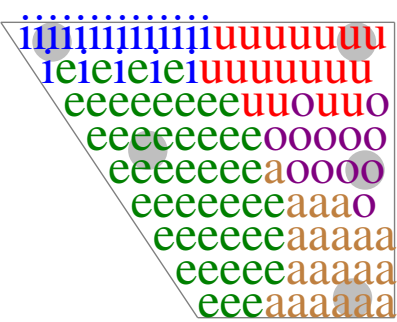

Acoustic $F_{1}, F_{2}$ space. Humans judging artificial vowels. Colours/letters show perceptual regions. Grey blobs show areas judged 'best' representatives of the five vowels.

Fig. 6. Perception of Spanish and Czech vowels, adapted from Savela 2009
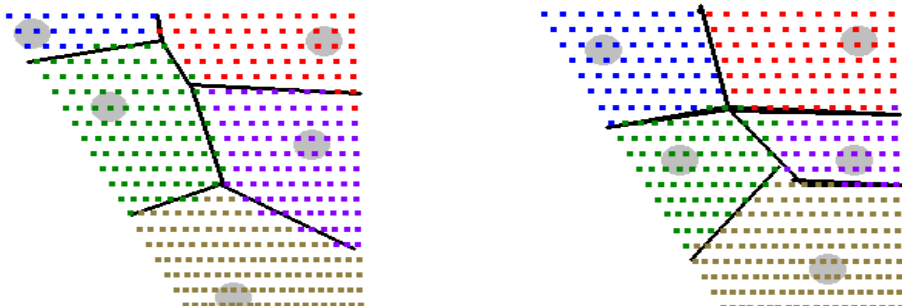

Agents learning from initial vowel inventories marked by blobs. Shades map perceptual boundaries of a new adult after a few generations.

Fig. 7. Agent simulation of vowel systems

It is immediately noticeable that the rather small difference in the positions of the prototypes has a noticeable effect on the perceptual regions that agents acquire; and that in particular, the apparent slope of some region boundaries is changed from fairly diagonal to fairly horizontal; in general, the shape of these boundaries is not clearly identifiable as more similar to simple prototype-learning predictions than simple feature-learning predictions; and that the results match the empirical results of Savela 2009 rather well, particularly given the purely geometrical notion of vowel I use.

I have to admit that these results surprised me. The prototype-and-perceptual-region model is sufficiently complex that a simple qualitative analysis is not obvious, but my intuition was that the perceptual polygons would approximate circles, and the perceptual boundaries would end up in much the same place as before.

To summarize, I have here argued that two simulations with contrary intents give similar results, so that the original simulation does not itself argue strongly for feature-based perception. Of course, there are other ways to argue for it, and recently Chládková, Boersma, and Benders (2015) have presented an elegant perception discrimination experiment which provides direct evidential support for perception of features, so vindicating their original contention.

\section{Discussion}

In the foregoing, I have looked at a sample of simulation work on a common theme, spanning forty years. I have suggested that in most of these, the simulations are actually adding little to the content of the work. With more space, I could have adduced many other examples. Of course, I am not alone in this view; in discussions at conferences and elsewhere, many have agreed with my scepticism. But what can simulations do? When are they useful? Why does phonology (or linguistics) have a problem, when simulations are so widely used in the rest of science? 
Firstly, what can simulations do? Most phonological simulations are designed to show that some theory or model could describe (or explain) some phenomenon. Simulations are very good at this: if you do a simulation of imitation-based learning, and end up with roughly human vowel systems, you can indeed conclude that imitation-based learning could be the reason for the shape of human vowel systems. So, however, could all the other uncountable many theories; and equally, countless variants of your pet system will not give the right results. It is rare to see a phonological simulation study that concludes that a particular range of models cannot account for a phenomenon, which would be a significant scientific contribution.

Part of the reason for this is obvious enough: the difference between the science underlying simulations in phonology and simulations in the physical sciences. In much of physical science, our model of reality is superbly accurate (quantum electrodynamics famously matches experiment to ten or twelve significant figures) and moreover our models appear to be 'real', in the sense that their structure corresponds exactly to the structure of reality - our fundamental models are both intensional and unabstracted, although we also know that they are wrong! In the physical sciences, simulations are used almost entirely to calculate things that simply cannot be calculated by analytic means. A typical example is weather, or even climate: though in principle we understand nature at the level of molecules and below, the complex and usually chaotic interactions of many entities make direct analysis utterly impossible. Simulations make very crude spherical cow-type abstractions - dividing the atmosphere into km-sized blocks but even so, the abstracted entities also have highly reliable models (ideal gas dynamics, with corrections), which in turn can be derived from and validated against the 'real' models. It is also the case that physical and biological scientists routinely use millions of times the computational resource available to linguists (e.g. millions of CPU-hours to simulate one microsecond of the motion of one molecule).

In more human applications, such as modelling traffic flows to solve congestion problems, the model is much cruder, whether it is differential equations (crude because continuous) or interacting agents (much less complex than real drivers). Nonetheless, the model can be validated against large amounts of observational data; and need not be accurate. Many stochastic simulations have $20 \%$ errors compared to the real system; but that may be good enough for the purpose at hand (for example, city traffic models done in my department).

In phonology, however, we have no equivalent of the Standard Model, or even of ideal gases. We have many competing models, none of which is evaluable with precise numerical rigour against reality, and none of which addresses more than a small part of phonology and phonetics. Even in small areas, if we try to make detailed models, we often end up with Cthulhucows, as De Boer did in his first attempt at his work.

What can we get from simulations? Possibility demonstration is not useless: if the model is modestly complex, the fact that it can describe or account for some phenomenon may not be clear. An example of this is the demonstration in section 4.2 that a non-feature learning system with a slightly richer vowel model can match data as well as a feature-learning system.

To make stronger statements, it is necessary to spend more time and effort on analysis of theories and methodologies. A recent example is Kirby 2010, which studies the effect of phonetic cues on phonological (re-)categorization, and is notable for using real acoustic data, as well as for a fairly sophisticated representation. Another is Sóskuthy 2013, which studies the notoriously difficult actuation problem, considering the interplay of phonetic biases with categorical effects, and conducts an unusually detailed analyis both of the underlying theories and the expected accuracy of the simulations. Nonetheless, even these careful studies do not produce strongly constraining results.

To repeat my thesis from the introduction, if simulations are designed with careful analysis 
of the underlying theories, analyses of the sources of error, and the rest of the apparatus usual in physical and engineering science simulation studies, and then simulations are conducted over a wide range of possible configurations and parameter settings, one might say with some confidence what is not possible, as well as what is possible; and one may even produce numerical results, testable for agreement with real empirical data.

$Q$ : How does a phonologist hear a cow? A: Consider a binary feature vector ...

\section{References}

De Boer, Bart (1999). Self-Organisation in Vowel Systems. Ph. D. thesis, Vrije Universiteit Brussel.

De Boer, Bart (2001). The Origins of Vowel Systems. Oxford University Press.

Boersma, Paul and Kateřina Chládková (2010). Perceptual difference in five-vowel systems reflect differences in feature structure. Presentation at the 18th Manchester Phonology Meeting.

Chládková, Kateřina, Paola Escudero, and Paul Boersma (2008). A cross-dialect acoustic description of vowels: Peruvian versus European Spanish. Poster presented at the Acoustic Society of America 2008 meeting.

Chládková, Kateřina, Paul Boersma, and Titia Benders (2015). The perceptual basis of the feature vowel height. In Proc. XVIII'th International Congress of Phonetic Sciences. abstract no. 711.

Donegan, Patricia J. (2004, January). Review of Bart de Boer, The Origins of Vowel Systems. J. International Phonetic Association 34(1), 95-100.

Ekštein, Kamil (2004). Hybrid Methods of Acoustic-Phonetic Analysis of Spontaneous Speech. Ph. D. thesis, University of West Bohemia in Pilsen.

Frigg, Roman and Stephan Hartmann (2017). Models in science. In Zalta, Edward N. (Ed.), The Stanford Encyclopedia of Philosophy (Spring 2017 ed.). Metaphysics Research Lab, Stanford University.

Harmegnes, Bernard and Dolors Poch-Olivé (1992). A study of style-induced vowel variability: Laboratory versus spontaneous speech in Spanish. Speech Communication 11, 429-437.

Ingerman, Peter Zilahy (1967). "Pạnini-Backus Form" suggested. Communications of the ACM 10(3), 137.

Johnson, Keith (2005). Speaker normalization in speech perception. In Pisoni, David and Robert Remez (Eds.), The Handbook of Speech Perception, pp. 363-389. Wiley-Blackwell.

Karttunen, Lauri (2006). The insufficiency of paper-and-pencil linguistics: the case of Finnish prosody. In Butt, Miriam, Mary Dalrymple, and Tracy Holloway King (Eds.), In Intelligent Linguistic Architectures: Variations on themes by Ronald M. Kaplan, pp. 287-300. CSLI Publications.

Kirby, James (2010). Cue Selection and Category Restructuring in Sound Change. Ph. D. thesis, University of Chicago.

Klein, Sheldon (1966). Historical change in language using Monte Carlo techniques. Mechanical Translation 9(3 and 4), 67-82.

Liljencrants, Johan and Björn Lindblom (1972, December). Numerical simulation of vowel quality systems: The role of perceptual contrast. Language 48(4), 839-862.

Lindblom, Björn and Johan Sundberg (1969). A quantitative model of vowel production and the distinctive features of Swedish vowels. STP-QPSR 10(1), 14-32.

Marr, David (1977). Artificial Intelligence: A personal view. Artificial Intelligence 9(September), 37-48. 
Pierrehumbert, Janet (2002). Word-specific phonetics. In Laboratory Phonology VII, pp. 101139. Mouton de Gruyter.

Prince, Alan and Paul Smolensky (1993). Optimality theory: Constraint interaction in generative grammar. Technical Report 2, Rutgers University Center for Cognitive Science.

Savela, Janne (2009). Role of Selected Spectral Attributes in the Perception of Synthetic Vowels. Ph. D. thesis, University of Turku.

Steels, Luc (1997). The synthetic modelling of language origins. Evolution of Communication 1(1), 1-35.

Stevens, Kenneth N. (1952). The perception of vowel formants. J. Acoustic Society of America 450, 450. Abstract of presentation at ASA meeting.

Sóskuthy, Marton (2013). Phonetic Biases and Systemic Effects in the Actuation of Sound Change. $\mathrm{Ph}$. D. thesis, University of Edinburgh.

Volín, Jan and David Studenovský (2007). Normalization of Czech vowels from continuous read texts. Paper presented at the XVI'th International Congress of Phonetic Sciences. 